\title{
Methotrexate-Associated Lymphoma
}

National Cancer Institute

\section{Source}

National Cancer Institute. Methotrexate-Associated Lymphoma. NCI Thesaurus. Code C150678.

A non-Hodgkin or Hodgkin lymphoma that develops in patients who are immunosuppressed with methotrexate. 